GESCHICHTE DES DEUTSCHEN FILMS 


\section{GESCHICHTE DES DEUTSCHEN FILMS}

Herausgegeben von

Wolfgang Jacobsen, Anton Kaes und Hans Helmut Prinzler

in Zusammenarbeit mit der Stiftung Deutsche Kinemathek Berlin 
Die Deutsche Bibliothek-

CIP-Einheitsaufnahme

Geschichte des deutschen Films/hrsg. von Wolfgang Jacobsen

... in Zusammenarbeit mit der Stiftung

Deutsche Kinemathek Berlin. -

Stuttgart; Weimar: Metzler, 1993

ISBN 978-3-476-00883-1

NE: Jacobsen, Wolfgang [Hrsg.]

ISBN 978-3-476-00883-1

ISBN 978-3-476-02918-8 (eBook)

DOI 10.1007/978-3-476-02918-8

Dieses Werk einschließlich aller seiner

Teile ist urheberrechtlich geschützt.

Jede Verwertung außerhalb der engen

Grenzen des Urheberrechtsgesetzes

ist ohne Zustimmung des Verlages

unzulässig und strafbar. Das gilt insbe-

sondere für Vervielfältigungen, Über-

setzungen, Mikroverfilmungen und

die Einspeicherung und Verarbeitung

in elektronischen Systemen.

(C) 1993 Springer-Verlag GmbH Deutschland

Ursprünglich erschienen bei J. B. Metzlersche Verlagsbuchhandlung

und Carl Ernst Poeschel Verlag GmbH in Stuttgart 1993 


\section{INHALT}

Vorwort

Von Wolfgang Jacobsen, Anton Kaes, Hans Helmut Prinzler

Frühgeschichte des deutschen Films

Von Wolfgang Jacobsen

Film in der Weimarer Republik

Von Anton Kaes

Exilfilm, 1933-1945

Von Jan-Christopher Horak

101

Film im Nationalsozialismus

Von Karsten Witte

Westdeutscher Nachkriegsfilm

Von Fritz Göttler

171

Film der sechziger Jahre

Von Norbert Grob

Film der siebziger Jahre

Von Claudia Lenssen

Film der achtziger Jahre

Von Eric Rentschler

Film in der DDR

Von Wolfgang Gersch

New Look: 13 filmische Momente

Von Frieda Grafe

365

Dokumentarfilm, 1892-1992

Von Klaus Kreimeier

391

Experimentalfilm, 1920-1990

Von Christine Noll Brinckmann

417

Filmkritik und Filmtheorie

Von Helmut H. Diederichs

451

Ein feministischer Blick

Von Heide Schlüpmann

Filmzensur und Selbstkontrolle

Von Martin Loiperdinger 
Inhalt

499

519

559

578

579
Fernsehen und Film

Von Karl Prümm

Chronik, 1895-1993

Von Hans Helmut Prinzler

Bibliografie

Autoren, Abbildungen, Dank

Register 


\section{VORWORT}

\section{Gedanken und Fragen}

Von Wolfgang Jacobsen, Anton Kaes und Hans Helmut Prinzler

1. 1995 wird der Film 100 Jahre alt. Er hat in einer unvorhersehbaren Intensität auf das 20. Jahrhundert eingewirkt. Sein Einfluß auf die Menschen, auf ihre Phantasien, ihre 'Träume und ihre psychische Realität war größer als der jeder anderen Kunst, und Kunst war auch nur der geringste Teil seiner Intentionen. Sein Warencharakter, seine technische Reproduzierbarkeit und seine ideologische Funktionalität haben den Film zu einer Produktivkraft der unterschiedlichsten Gesellschaftssysteme in diesem Jahrhundert gemacht. In Deutschland waren dies: eine Monarchie (bis 1918), eine erste parlamentarische Demokratie (1918-1933), eine faschistische Diktatur (1933-1945), eine sozialistische Diktatur im Osten (1945-1989) und - zur gleichen Zeit - eine zweite parlamentarische Demokratie im Westen, die seit 1990 für eine neue Bundesrepublik gilt. Eine Geschichte des deutschen Films muß im Kontext dieser historischen Entwicklung und dieser Gesellschaftssysteme geschrieben werden.

2.

Andernorts - in Frankreich etwa, in Großbritannien, Italien und natürlich in den USA - sind nationale Filmgeschichten längst geschrieben, und je nach Bedarf werden ihnen neue Kapitel hinzugefügt. Ihnen allen gemeinsam ist, daß sie sich an der Chronik der laufenden Ereignisse orientieren; sie nähern sich - der Gesamtdarstellung verpflichtet - nur selten über Einzelaspekte der Filmgeschichte. Sie entwerfen einen generellen, gelegentlich auch generalisierenden Überblick. $\mathrm{Na-}$ türlich schränkt die notwendige Verknappung einen detaillierten analytischen Diskurs ein.

Über einzelne Phasen der deutschen Filmgeschichte gibt es kompetente Darstellungen. Sie haben sich aber nicht zu einer Gesamtsicht gefügt. Das Schreiben über die deutsche Filmgeschichte ist Arbeit an einer $>$ Bausteller. Im November 1968 konnte man in den >Filmkundlichen Mitteilungen< des Deutschen Instituts für Filmkunde lesen: »Eine Geschichte des deutschen Films - sie ist das vielleicht wichtigste Unternehmen der noch in Ansätzen befindlichen aktuellen Filmforschung. « Nun - 25 Jahre später - ein erster zusammenhängender Blick auf die deutsche Filmgeschichte. Ohne sich dem Zwang zur Vollständigkeit zu unterwerfen. Es geht uns vielmehr um Zusammenhänge, offene und versteckte Verbindungslinien, rote und graue Fäden. Nicht nur die deutsche Produktion liegt im Blickfeld, sondern auch die Rezeption in Deutschland. So werden Stiller, Chaplin, Eisenstein und Godard Teil einer deutschen Filmgeschichte.

Die Sicht der Auroren, auch wenn sie auf fast 100 Jahre deutschen Film konzentriert ist, grenzt ihren Gegenstand nicht nationalpolitisch ein. Im Gegenteil: Sie öffnet, so hoffen wir, den Blick für Zusammenhänge, die jenseits von Landeskoordinaten und vor allem jenseits von nationalen Empfindungen bestehen. Filmgeschichte, auch die deutsche, ist nur zu erzählen in ihren internationalen Verknüpfungen, die
"Wenn mein Herz gesund wär, spräng ich zuerst aus dem Fenster; dann ging ich in den Kientopp und käm nie wieder heraus." (Else Lasker-Schüler: Kinematographisches. In: Der Sturm, April 1912).

"Der Film ist verbreiteter als das Theater, besuchter als das Theater. Infolgedessen soll man die größte Aufmerksamkeit auf den Film legen. Möglicherweise gehört er mehr unter die Schnapssorten als unter die Kulturprodukte. Aber auch die Syphilis ließ sich durch Ignorierung nicht an Ausdehnung beirren; die Krankenkassen, die Geschlechtskrankheiten früher beinah bestraften, zahlen jetzt sorgfältig Krankengeld; man drängt sogar auf Behandlungszwang."

(Alfred Döblin: Europäische Krise, Gesang, Film. In: Prager Tageblatt, 28. 4. 1922). 
geradezu identitätsbestimmend sind. Das schließt nicht aus, daß zur deutschen Filmgeschichte Eigenheit und Eigensinn gehören. Man denke nur an die prekäre Ambivalenz von Film-Kunst und Film-Politik. Oder an den Antagonismus von Industrie und Kunst.

3.

»Wir leben erst im Anfangsstadium des Films, und die Eroberungen, die das Kino machen wird, werden sich mit ungeheurer Schnelligkeit vermehren und ungeahnte Dimensionen annehmen. An dieser Tatsache ist nicht zu rütteln, darüber sollten sich alle Kinogegner klar sein und ihre Kraft nicht unnütz gegen das Kino verschwenden. Was wollen diese Leute überhaupt vom Kino? Kunst wollen sie vom Kino; da der Film keine Kunst sei, habe er keinerlei Existenzberechtigung. Diese Auffassung wird nicht nur von verknöcherten Jugendbildnern und Konsorten vertreten, sondern auch ernsthafte Künstler und Schriftsteller nehmen diesen Standpunkt ein. Ich werfe daher die Frage auf: >Muß der Film, um den Beweis seiner Existenzberechtigung zu erbringen, Kunst sein?c, und muß diese Frage verneinen. Es ist sehr schön und auch ein Ziel aufs innigste zu wünschen, daß der Film, oder wenigstens ein Teil der Filmproduktion eine künstlerische Höhe erreicht; von der Filmproduktion im allgemeinen aber kann man es nicht und braucht man es nicht zu verlangen. «

(Ernst Lubitsch: Uns fehlen Filmdichtungen. In: Das Tagebuch, 11. 9. 1920).

4.

»Die Burg Etzels ist abgebrannt. Das geschah in Neubabelsberg, wo der Regisseur Lang die Aufnahme für den zweiten Teil des Nibelungenfilms mit eben jenem Brand vollendet hat. Man weiß, daß der Regisseur Lang am Tage der Uraufführung des Nibelungenfilms einen Pietätskranz am Grabe Friedrichs des Großen niedergelegt hat. Nun bin ich neugierig, welches Königsgrab er bei der Uraufführung des zweiten Teils zu schmücken beabsichtigt.

Herrlich war der Brand in Neubabelsberg. 400 Leute waren eingeladen. Feuerwehr und Schutzpolizei sperrten den Platz. Der Brand rötete den nächtlichen Himmel phänomenal. In dem Gewimmel behielt der Regisseur Lang Geistesgegenwart und Monokel.

Mitten aus den Trümmern (altes Leben, aus den Ruinen blühend) tauchte eine deutsche Frauengestalt auf, mit dem Vornamen s Thear.» (Joseph Roth: Berliner Bilderbuch. In: Der Drache, 1. 4. 1924).
17 Autorinnen und Autoren haben zu dieser 'Geschichte des deutschen Films< beigetragen. Alle - im besten Sinne - mit einer eigenen Handschrift. Und damit ist auch fast schon die Frage nach dem methodischen Selbstverständnis beantwortet, die sich für die Herausgeber jeder Filmgeschichte stellt. Die meisten unserer Autorinnen und Autoren sind Spezialisten für ihren Themenbereich. Sie bringen Kontinuität in der Beschäftigung mit einem Genre oder einem Zeitraum deutscher Filmgeschichte ein. Einige betreten Neuland, nähern sich so ausführlich und umfassend - erstmals ihrem gewählten Gegenstand. Das Interesse für ihr Thema haben sie aber schon in früheren Veröffentlichungen angedeutet. Viele Autorinnen und Autoren zeichnet zusätzlich aus, daß sie im Denken, in der Analyse und Bewertung Grenzgänger sind. Sie knüpfen Verbindungen zwischen dem Film und den anderen Künsten, suchen etwa nach Berührungspunkten oder Beeinflussungen im Bereich der Literatur und Musik, der Architektur und Malerei, der Philosophie und Alltagsgeschichte; nicht zuletzt spielt in ihren Überlegungen auch das historisch-politische Moment eine Rolle. Filmgeschichte ist nicht unabhängig von sozialen und politischen Ereignissen zu interpretieren.

5.

1992 haben die Autorinnen und Autoren dieser , Geschichte des deutschen Films ihre Texte geschrieben. Es war ein Jahr der Gedenktage und der filmhistorischen Jubiläen. Im Januar wurde der 100. Geburtstag von Ernst Lubitsch gefeiert. Das Filmstudio Babelsberg existierte im Februar 80 Jahre. Im April jährte sich zum 30. Mal der Tag der Unterzeichnung des >Oberhausener Manifests`. Im Mai starb Marlene 
Dietrich, 90 Jahre alt. Im Juni wurde des zehnten Todestages von Rainer Werner Fassbinder gedacht. Die Münchner >Hochschule für Fernsehen und Film< feierte im November ihren 25. Geburtstag. Und im Dezember stand die berühmteste deutsche Filmfirma im Blickpunkt: 75 Jahre Ufa.

Mit Retrospektiven, Ausstellungen und Publikationen, mit Gedenksendungen, Nachrufen und Festreden wurden die Geburtstage, Todestage und Jubiläen gewürdigt. Zeichen für ein generell gewachsenes Interesse an Filmgeschichte?

6.

»Jahrelang war Deutschland von der übrigen Welt abgeschlossen. (Ist es noch, könnte man beinahe sagen.) Nirgends werden die furchtbaren Folgen deutlicher als im Kino. Man vergleiche einmal Leo Peukert mit Charlie Chaplin, Bruno Kastner mit Douglas Fairbanks, Mia May mit Mary Pickford - und man hat den Unterschied - nicht zwischen deutschem und amerikanischem Film, sondern zwischen Deutschland und der übrigen Welt. Gewiß gibt es bessere Leute im deutschen Film als Peukert und Kastner. Asta Nielsen, Jannings, Abel, Wegener - das sind schon Leute, die sich überall in der Welt sehen lassen können. Aber der Geist, den sie vertreten müssen, der Geist, die Gesinnung, die Art, der Stil des deutschen Films, der kann sich nicht sehen lassen. (...) Kaiserwilhelmgedächtniskirche - das ist der äußere Bau des deutschen Films. Kaiserparade - das ist der Inhalt.

Und während dessen wackelt Charlot, ein weiser und gütiger Vagabund, an allen blamablen Abgründen des Lebens entlang - komisch, rührend und nichts anderes als ein Mensch. Ganz ohne Pomposo und ohne Zement und ohne Kultur- und andere Historie. Ganz einfach und alltäglich.

Am deutschen Wesen soll die Welt genesen? Das möge Gott verhüten!«

(Hans Siemsen: Deutsches Kino. In: Der Querschnitt, Weihnachtsheft 1922).

\section{7.}

Einige spezifische Genres und Subgenres des deutschen Films, geordnet nach dem Zufall des Alphabets: Arztfilm, Aufklärungsfilm, Bergfilm, Biografischer Film, Edgar Wallace-Film, Expressionistischer Film, Heimatfilm, Karl May-Film, Kinderfilm, Kriegsfilm, Kriminalfilm, Kulturfilm, Literaturverfilmung, Lustspiel, Melodram, Preußenfilm, Problemfilm, Revuefilm, Schlagerfilm, Sexfilm, Straßenfilm.

Über die Nachbarschaften ist nachzudenken. Und: Was hat der Preußenfilm mit dem Revuefilm zu tun, der Arztfilm mit dem Melodram, der Kulturfilm mit dem Sexfilm? Die Frage nach spezifisch deutschen Zusammenhängen.

8.

International ist ein neues Interesse an Filmgeschichte seit Anfang der achtziger Jahre festzustellen, das gewachsen ist mit dem Stummfilmfestival im italienischen Pordenone. Indizien dafür waren auch: das Erscheinen der ersten theoretischen Abhandlung über Probleme der Filmgeschichtsschreibung von Robert C. Allen und Douglas Gomery (1985); die Veröffentlichung historisch-faktisch orientierter Studien
»Nieder mit Fridericus, rettet Otto!!

(Carl Otto Graetz: Rettet Gebühr... In: Das Tagebuch, 18. 6. 1927).
»Woher die leeren Theater? Nur durch das Ausbleiben des Publikums. Schuld daran - nur der Staat. Warum wird kein Theaterzwang eingeführt? « (Karl Valentin: Zwangsvorstellung. 1932). 
»Uns trennt von gestern kein Abgrund, sondern die veränderte Lage.«

(Schrifttitel in Alexander Kluges ABSCHIED VON GESTERN, 1965/66). wie 'Film Style and Technology: History and Analysis < von Barry Salt (1983) und The Classic Hollywood Cinema. Film Style and Mode of Production to 1960, von David Bordwell, Kristin Thompson und Janet Steiger (1985). Filmwissenschaftler wie Thomas Elsaesser, Miriam Hansen und Patrice Petro versuchten, Erkenntnisse der psychoanalytischen Filmtheorie mit neuen historischen Fragen zu verbinden. In der Bundesrepublik beschäftigte sich 1988 eine Tagung der >Gesellschaft für Film- und Fernsehforschung mit Entwürfen und Methoden der Filmgeschichtsschreibung. Seit 1984 liefert das Lexikon >CineGraph schen Filmgeschichte, seit 1988 findet in Hamburg jährlich ein filmhistorischer Kongreß statt. Ist Filmgeschichte in Mode gekommen?

9.

Deutsche Regisseure, herausragende Namen: Lubitsch, Lang, Murnau, Pabst, Ophüls, Siodmak, Riefenstahl, Harlan, Käutner, Staudte, Wolf, Fassbinder, Wenders.

Andere Namen, deren Wiederentdeckung gerade begonnen hat oder noch aussteht: Stellan Rye, Urban Gad, Joe May, Richard Oswald, E. A. Dupont, Ludwig Berger, Karl Grune, Arthur Robison, Werner Hochbaum, Herbert Selpin, Rudolf Jugert, Gerhard Klein.

Was haben sie, außer in dieser chronologischen Aufzählung, für eine Verbindung untereinander? Beziehen sich die Späten auf die Frühen? Die Frage von Kontinuität, Tradition und Beeinflussung wird im deutschen Film primär politisch gestellt.

10.

"Als der Krieg glücklich verloren war, da war das eigene Nest hoffnungslos verdreckt von oben bis unten. Und da kein revolutionäres Großreinemachen stattfand, wurde der Dreck versteckt, so gut es ging, aber er blieb im eigenen Nest...

Die aber, die sich diese Zeit dennoch nahmen und den Dreck aus den eigenen Ecken hervorzukehren versuchten, es waren gottlob nicht wenige, mußten oft auf den Dank des Vaterlandes verzichten und erfahren, daß ihr politisches Reinlichkeitsbedürfnis eben von jenem Kommentar begleitet wurde, der der Anlaß zu diesen Zeilen ist: Der beschmutzt ja sein eigenes Nest! (...)

Politische Filme sind ein Stück Geschichtsdarstellung der Gegenwart. Sofern sie Kunst sind, werden sie parteiisch sein, herausfordernd und subjektiv, aber immer anteilnehmend und besorgt um den $\mathrm{Zu}-$ stand des reigenen Nestes<. (...)

Sicher sind es nicht die Filme, die das eigene Nest beschmutzen."

(Wolfgang Staudte: Das eigene Nest beschmutzen? 1964).

11.

"Was ist das Deutsche am deutschen Film? «, fragt Frieda Grafe. "Das Vaterländische, das Muttersprachliche, das Einheimische? Jedenfalls nicht die Nationalität. Die Finanzierung, das Sujet, der Drehort, der Tobisklangfilmton? Eher noch, was öffentlicher Meinung entspricht, wobei die Phantasien der Ausländer abweichen vom deutschen Selbstverständnis. "So schreibt sie über Carl Mayer und Carl Theodor Dreyer, über Reinhardt und Sternberg, über Syberberg und Rossellini, über Kluge und Straub. Das Eigene im Fremden entdecken - und das 
Fremde im Eigenen. Und das Nationale als Fälschung entlarven, als Tünche über dem eigentlichen Bild. Alles ist eine Frage nicht der Identität, sondern der Identitäten. Insofern ist eine deutsche Filmgeschichte ein sinnvolles Paradoxon - zu spät und zu früh.

12.

Bilder von Guido Seeber, Karl Freund, Robert Baberske, Eugen Schüfftan, Carl Hoffmann, Fritz Arno Wagner, Günther Anders, Igor Oberberg, Friedel Behn-Grund, Werner Bergmann, Richard Angst, Robby Müller, Martin Schäfer, Jörg Schmidt-Reitwein, Gernot Roll, Michael Ballhaus und vielen anderen. Es ist erstaunlich, daß es kein Buch über deutsche Kameraleute gibt.

Über die eigentlichen >Bilder< darf man die Architektur nicht aus dem Auge verlieren, die Filmarchitektur, eine Stärke des deutschen Filmhandwerks: Walter Röhrig, Robert Herlth, Emil Hasler, Otto Hunte, Erich Kettelhut, Herbert Kirchhoff, Willy Schiller, Alfred Hirschmeier, Rolf Zehetbauer, Heidi und Toni Lüdi. Auch ihre Arbeit ist Arbeit an Bildern.

Bilder werden auch geschrieben. In jüngster Zeit gibt es eine Debatte über die "Spezialisten einer Industrie«, die Drehbuchautoren; von der Wiederentdeckung des Drehbuchs ist die Rede. Und dabei auch von Carl Mayer - dem literarischen Drehbuchautor; von Willy Haas dem Literaten als Drehbuchautor. Andere Namen sind: Luise Heilborn-Körbitz für das Triviale in der Weimarer Republik; Thea von Harbou für die Kunst im Trivialen und das unheimlich Triviale in der Politik. Oder Axel Eggebrecht, ein »zwiespältiger Fall « in seinem Engagement im Dritten Reich - wie Karsten Witte anmerkt. Die Namensreihe ist fortzusetzen bis zu Wolfgang Kohlhaase, dem Geschichtenerzähler, und Wolfgang Menge, dem anderen, der Geschichte in Geschichten erzählt.

$\mathrm{Zu}$ reden und zu schreiben ist auch über die Töne, die Musik, die zu Bildern gemacht wird oder die Bilder oft erst zu Bildern macht. Filmkomponisten haben schon im stummen Film etwas zu ssagen< gehabt. Im Tonfilm sind sie dann groß geworden: Giuseppe Becce - der musikalische Grandseigneur, Werner Richard Heymann - der pointierte Illustrator, Herbert Windt - der raffinierte Propagandist, Peer Raben der gewiefte Tonmaler.

Das Imaginäre entsteht im Zusammenspiel verschiedener Kräfte. Das ist kreative Teamarbeit und Film als Gesamtkunstwerk: "Ihrer wahren Natur gemäß sollte die Filmkunst eine vollständige Bildgeschichte erzählen «, schreibt der Regisseur Friedrich Wilhelm Murnau im Jahr 1927.

13.

"Vielleicht wichtigstes Kennzeichen des deutschen Films - und zugleich jenes, das ihn am meisten abhebt vom Film anderer Länder - ist seine Nähe zum Theater. Alle bedeutenden Theaterschauspieler - Jannings, Krauß, Kortner, Bergner, Wessely - haben gefilmt. Alle bedeutenden Theaterregisseure haben auch Filme gedreht. So kommt es, daß deutsche Filme von Beginn der Tonfilmzeit bis in die sechziger Jahre konzipiert wurden wie Theaterstücke.«

(Ulrich Kurowski: Was ist ein deutscher Film? Mutmaßungen über eine wenig bekannte Sache. In: Film-Korrespondenz, 2. 11. 1973).
"Wünschenswert erschiene mir heute eine Filmgeschichte, die überhaupt nicht chronologisch, horizontal vorginge, sondern vertikal, die die über achtzig Jahre verstreuten Erscheinungen wie gleichzeitige ansähe und ihre historischen Schichten und deren Verwerfungen aufdeckte.

(Enno Patalas: Zehn Jahre später. In: Ulrich Gregor, Enno Patalas: Geschichte des Films. München, Gütersloh, Wien 1973).

"Die Geschichte des Films ist der einzig sichtbare Teil der Geschichte, und in diesem Sinne ist es die Weltgeschichte, die zur Filmgeschichte gehört. « (Jean-Luc Godard: Einführung in eine wahre Geschichte des Kinos. München, Wien 1981). 
"Als ich Kind war, habe ich mich oft gefrag $\iota$, ob es wirklich einen lieben Gott gibt, der alles sieht. Und wie er es dann schafft, nichts zu vergessen: weder die Bewegung der Wolke am Himmel noch all die Gesten und Schritte von einem Jeden, die Träume jedes Menschen... Ich sagte mir: Unmöglich, sich vorzustellen, daß ein solches Gedächtnis existiert, und noch trauriger und unmöglicher zu denken, daß es nicht existiert, daß alles in Vergessenheit gerät. Diese kindliche Vorstellung beunruhigt mich noch immer. Unendlich groß wäre also die Geschichte der Erscheinungen, unendlich klein die der Bilder, die überleben.

Während Hunderten von Jahren haben einzig die Dichter und die Maler sich mit dieser gigantischen Gedächtnisarbeit beschäftigt. Dann haben die Fotografen einen erheblichen Beitrag geleistet, dann die Filmleute, mit immer größeren Mitteln und immer weniger gutem Willen. Heutzutage schließlich ist es vor allem das Fernsehen, das die Bilder bewahrt. Aber in der Inflation elektronischer Bilder, die das Fernsehen uns bietet, scheint in einem solchen Maße wenig Erinnerungswürdiges zu sein, daß man sich fragen muß, ob man nicht lieber zur alten Tradition der Dichter und Maler zurückkehren sollte. Es ist besser, wenig Bilder, aber voller Leben als Unmassen von sinnlosen Bildern zu haben.

Es ist der Blick, der darüber entscheidet, ob etwas gesehen worden ist."

(Wim Wenders: Eine Geschichte des imaginären Films. In: Cahiers du cinéma, Nr. 400, Oktober 1987).
14.

Filmgeschichte schreiben an einem chronologischen Faden entlang. Das ist Programm des ersten Teils dieses Buches. Es bietet den Lesern Stationen, an denen sie anhalten können. Sich orientieren. Bilder finden und sortieren. Sich an Filme erinnern, an Geschichten und Augenblicke. Die Chronologie ergibt keine Abfolge von Namen und Filmtiteln. (Das leistet auf eine andere Weise die Chronik im Anhang.) Sie liefert den Hintergrund, der die Produktions- und Rezeptionsgeschichte verständlich macht. Der erste 'Teil also: Längsschnitte. Der zweite: Querschnitte. Dokumentarfilm, Experimentalfilm, Kritik und Theorie, der feministische Blick, Zensur, Film und Fernsehen. Über die Totalen wollten wir die Naheinstellungen nicht vergessen. Die Desiderata, die >weißen Fleckes, kennen wir: Kinogeschichte, Technikgeschichte, Wirtschaftsgeschichte, Schauspielergeschichte. In Momenten sind diese Aspekte in den einzelnen Kapiteln dennoch aufgehoben. Und die Namen und Titel, die fehlen... In der ersten Fassung war uns das Buch auf über 900 Seiten explodiert.

Neben den Essays steht eine Marginalspalte. Sie liefert Querverweise und ergänzende Zitate; sie ist Assoziationsfeld - für gedankliche Schleichwege.

15.

"Es gibt keine Filmgeschichte. Es gibt eine Produktionsgeschichte, und es gibt Filme. Die Produktionsgeschichte kann erforscht, beschrieben und revidiert werden. Die Filme widersetzen sich der historisierenden Rezeption. Sie sind die einzigen Bilder, die dem kollektiven Gedächtnis des Zuschauers im musée imaginaire gehören.«

(Karsten Witte: Wie Filmgeschichte schreiben? In: Film und Fernsehen in Forschung und Lehre, Nr. 5, 1982)

16.

Warum wir den Film lieben oder das Gedächtnis der Welt.

Als Leser haben wir uns Entdeckungsreisende vorgestellt, die das Buch als sLandkarte benutzen und ihre Wegmarkierungen notieren, besser: ihre Seh-Erfahrungen. So kann eine »unendlich verwobene Fläche« - wie Robert Musil einmal schreibt - entstehen; Bilderfelder: für sich allein und sich überlagernd. Kreuzungspunkte werden sich ergeben. Und Exkursionen sind willkommen.

Diese >Geschichte des deutschen Films` versteht sich auch als >Bilder-Buch. Man kann sich vergucken - so oder so...

Berlin und Berkeley

März 1993 